\title{
Toward Semantic Mobile Web 2.0 through Multiagent Systems
}

\author{
Gonzalo A. Aranda-Corral, Joaquín Borrego-Díaz, and Fernando Gómez-Marín \\ Departmento Ciencias de la Computación en Inteligencia Artificial \\ Escuela Técnica Superior de Ingeniería Informática
}

Universidad de Sevilla

\begin{abstract}
In this paper, a solution for semantic annotation of Consumers Generated Content, based on the actions of a multiagent system, is presented. This solution is designed in the context of an spin-off which was born from Minerva project for mobile services 1 . Our proposal aims to build a Mobile Web 2.0 platform for the dissemination of interesting events, captured by users through mobile phones. The multiagent system creates an agent for each event, which suggests and manages several semantic features linked to an Ontology related to events.
\end{abstract}

\section{Introduction}

Nowadays, we observe a key convergence into the future of telecommunications. On the one hand, the great success of mobile networks implantation provide us with a global and ubiquitous net and, on the other hand, the exploding of social nets in Web 2.0 provides to users with tools for generating content of any kind. The convergence of the two global networks (see fig. 11(up), extracted of the book [4]) leads new kinds of personal communication and public sharing contents, example of mobile lifestreaming.

Despite this convergence -seems to be the "ultimate" convergence- more work on knowledge interoperability among heterogeneus devices (mobile phones, Web 2.0 mashups, Semantic Web or Intranets) will be needed. This convegence is called Metaweb in [10. As we will argue in section[2] the special features of mobile devices make hard the convergence of Mobile Web 2.0 with the Semantic Web. These may lead a new digital divide between Mobile Nets and Semantic Web or Intranets. This problem should be (at least partially) solved before massive user content generation.

In this paper, we want to show how to solve the problem for synthetizing sound metadata for user contents by means of using a multiagent system, which controls and advices the semantic annotation (see 1, down). In this way, the

Supported by the project 2C/040 WeTeVe of Minerva Platform of mobility services, http://www.proyectominerva.org/

A coordinated project of Andalusian Consejería de Innovación, Ciencia y Empresa, Vodafone, Universidad de Sevilla, Parque Científico y Tecnológico Cartuja'93, Sevilla Global S.A.M., ETICOM and AICIA. 


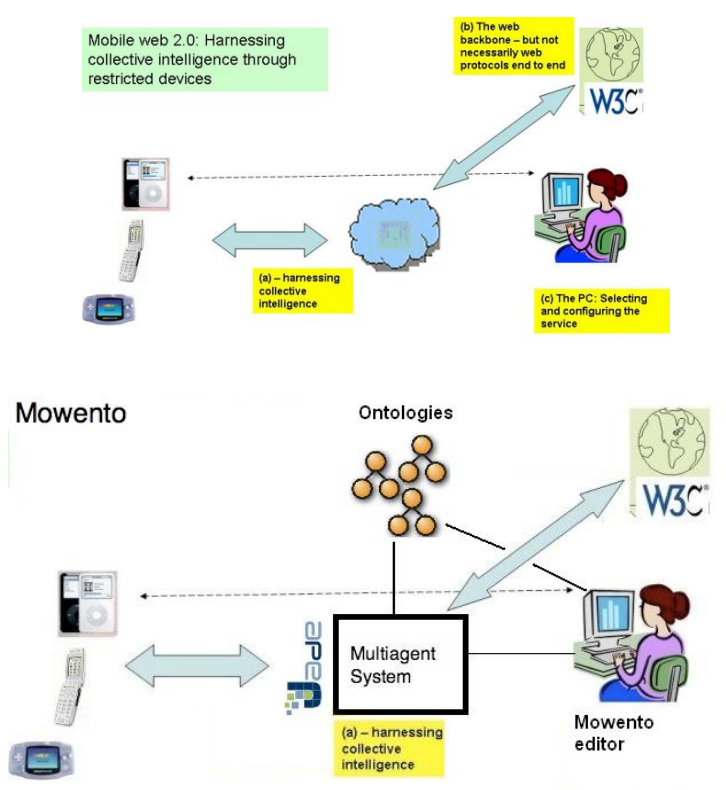

Fig. 1. Mobile Web 2.0(up) and Semantic Mobile Web 2.0 in Mowento (down)

system (called Mowento) bridges the gap between user tagging and annotation with respect to an ontology. Mowento is designed for testimonial documents publishing on events witnessed by the user at the time is going. These events are captured using mobile devices (photos and short videos). In section 4 we will describe this platform in more detail. These digital documents are sent to Mowento Platform, via MMS, to be published (in different ways, depending on user's account) into the WWW, as well as, an alert on this new event is broadcasted -via SMS- to Mowento neighborhood's user. The key engineering choice of Mowento is the use of a multiagent system (implemented on JADE2) to execute several semantic tasks on the documents. Each document, which is received in Mowento (via Vodaphone's Red Box platform), induces the creation of an agent associated to the document, and it will replace some of classical user Web 2.0 tasks. Here, we are focused on tagging, although there exists others semantic tasks, such as, visual ontology debugging inspired in [2].

\section{Features of Mobile Web 2.0}

"Mobile Web 2.0" (MW2.0) designation adresses to a new realm of platforms. Tools and social networking which share the main features of Web 2.0, but it also has specific characteristics. Some of these are related to mobile devices usability and user's behaviour. Briefly:

2 http://jade.tilab.com/ 


\subsection{Identity in MW2.0}

Users in Web 2.0 are identified by its avatar and its history into the social net. In case of mobile phones, users aim to achieve a similar identity, althoug its main nick is the phone number. Thus, MW2.0 platforms and tools, must to be designed to grant this requirement. An important example is the management of personal tags.

\subsection{Tagging and Mobile Devices}

Tagging is a social method to categorize or to classify documents. In Web 2.0, tagging is a task that different Web sites consider it in many different ways, mainly 9]:

- For managing personal information

- As social bookmarking

- To collect and to share digital objects

- For improving the e-commerce experience

In case of MW2.0, and specifically Mowento (which is designed for fast publishing of documents related on events), third motivation is very important. However, tagging from mobile devices can be a tedious task. Therefore, an ontology on events have to be used as a consensus ontology for tagging digital objects by a mobile application. User mobility is not considered as a key feature for ontological commitments, because such complex information can be provided by the system/platform (using geolocalization and post-tagging systems).

\subsection{Services in MW2.0}

MW2.0 services can be created in two different ways [6]:

- By mobile extension of an existing Web 2.0 service.

- By a pure MW2.0 service specifically dedicated to mobile network and based on user-generated content. New tools for generated knowledge management must be designed.

\subsection{Mobile Phones and Sharing Content}

Semantic annotations of digital documents from mobile devices have several limitations, some of them related to local features of telecomms:

1. Mobile phones are tedious tools for writing content. Thus, mobile applications should simplify tagging task. Nevertheless, this barrier turns on an advantage if mobile application shows us, as available tags, classes from Mowento's ontology. 


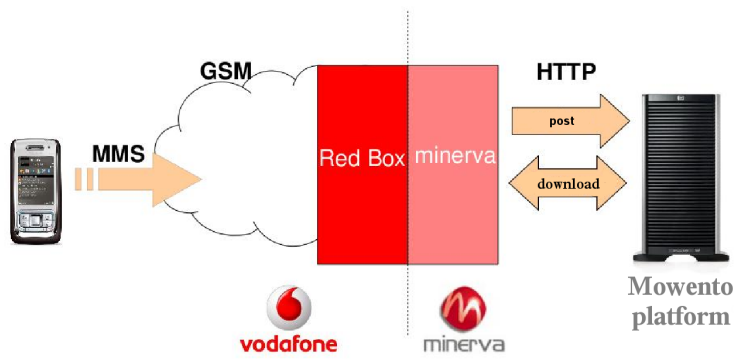

Fig. 2. MMS channel of Mowento

2. The user appreciate the inmediate generation of digital document on an event and its fast publication. This reason suggest the use of MMS channel instead of Mobile Internet (see 2). Moreover, in Spain, the relative higher cost of Mobile Internet services discourage this channel, if the application wants to have a great scope.

Regarding to document diffusion, MW2.0 platfoms must allow different levels of advertising and sharing, from private (own) use, personal use (shared with a trusted network), public, and even collaborative. That is, other users can add, transform or refine the information. Propagation through the WWW is a classic Web 2.0 service, enabled by WWW Mowento Platform, but distribution of this information in a mobile-based network is a more complex task than Web 2.0 8 . At mobile-based networks are important the weak ties because they connect social neighborhoods. Thus, a micro-dissemination of a document among the user's neighborhood ensures a greater impact than the publication in the WWW platform only. This decision is supported by the well-known thesis in Mobile Data Industry: the content is not the king, it is the contact [7]. In case of Mowento, the contact is the key bridge for micro-dissemination.

\section{Semantic Web and Mobile Web}

The convergence between Semantic Web and Mobile Web 2.0 depends on the specific management of ontologies. Ontologies and tags/folksonomies are two knowledge representation tools that must be reconciliated in metaweb projects. An useful bridge between these two kinds of representations could be Formal Context Analysis (FCA) [3]. FCA is a mathematical theory that formalizes the concept of "concept" and allow to compute concept hierarchies out of data tables, and it is also used for ontology mining from folksonomies [5].

In case of Mowento, FCA is used as an ontological representation of tags. Rougly speaking, ontology class $\mathrm{X}$, in Mowento's ontology, is intended as the class of digital objects with tags $X$. In fact, Mowento's ontology is extracted from several experiments with our mobile application and an arbitrary set of tags. Attribute exploration method is used to refine this ontology, as well as, to 
obtain a system for (post)suggesting tags. This decision was useful to solve the tedious task of tagging by means of mobile phones, because the ontology, that is offered in a mobile application, is an hierarchy of tags extracted from concept lattice, given by FCA. In this way, the interoperability among sets of tags from different users it is (partially) solved.

Nevertheless, it is necessary to augment and improve the set of tags ,by means of suggestion tags method, related to original user's tags. Mowento use the stem basis [3], associated to the ontology of tasks, as knowledge basis for a production system of suggested tags. These will be implemented as a new agent's behaviour, associated to the document at the platform (see section 51).

\section{Mowento's Services}

Mowento platform has been thought to facilitate publication of audiovisual documents and communication between members of a mobile social network. In this way, the main focus were to implement a fast and simple solution where generated content management should be as automatic as possible.

Some experimentation steps has been done previously, which they have been useful to define basic features to implement this initial stage. The solution that is described here (see fig. 3) can be divided into five different components, as follows.

\subsection{Mobile Application}

Mobile application is a key tool due to audiovisual documents are generated at the moment. So the user should find it attractive, fast and easy enough, to create and to send documents to the platform. Members joining on the network depends on the experience they have on these first steps, so mobile app will be decisive for the project success or failure.

Speed and simplicity are the main characteristics to be considered. The user of mobile device is not at a very comfortable environment to spend time writing a full description for the document, or doing complex steps which require special

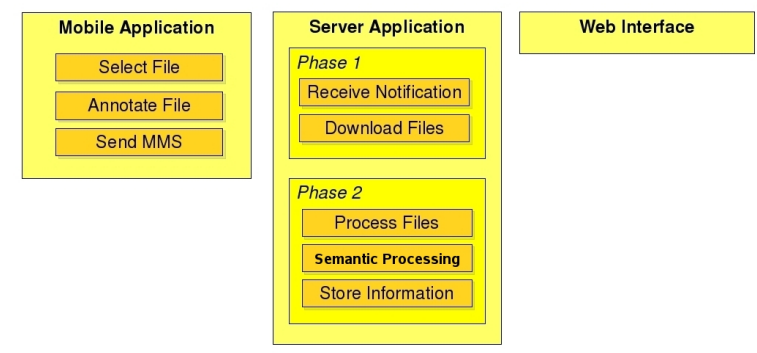

Fig. 3. Mowento service's structure 
attention. Basically, actions should be "Shot And Send" (SAS action). So a mobile application on this point is required.

There are a huge number of mobile devices types and due to one of principal aims of this application is to be accessible as much people as possible. For this, the first prototype was decided to be implemented in Java. It has been used J2ME, Java version for mobile platforms. It is the most extended language, in this kind of devices, and will permit us a fast development.

The features implemented for this first prototype have been the picking of the selected file, that could be a short video or a photograph, with the current limit of $300 \mathrm{~KB}$ due to MMS limitations. Any other kind of file have not been considered for this project. It is also implemented the basic annotation of the document. The fields, which are provided, are title, small description, tags set and date. Default values are available and empty values are admitted for a fast sending. Finally, sending the full audiovisual document by MMS.

\subsection{Server Application}

The next application which is needed, is on the server side, for automatic reception, management and organization of user's audiovisual documents, sent by MMS. Our first prototype developed is based on a traditional solution for web platforms called LAMP. We mean Linux as operative system (Ubuntu), Apache as a web server, MySQL as database and finally PHP for dynamic web pages. The LAMP system is a robust and tested enough by a huge community of developers in a great number of web projects, and also is open source and free. These features made it our choice. The full system run on a dedicated standalone server.Thanks to Minerva project, we have got access to the Vodafone's Red Box service for SMS/MMS handling. Minerva offers a simple interface to this service for research projects like ours.

The application server has two phases: First one is the MMS reception: each MMS sent to our platform is notified by Minerva using a POST message, by HTTP protocol. So when the server application receives the notification of a MMS, it captures all the related information in the message about that MMS. Using them, all of attached files from Minerva are downloaded to our platform by HTTP protocol, too.

Second one, is the downloaded file processing: all the information collected about the file is saved into the database, afterwards it makes the needed transformations to a suitable formats for its publication on the web -also creates photographs and videos' thumbnails- and translate from native video format to flash video format (FLV). For this first implementation, only the jpg format for images and $3 \mathrm{gp}$ for video have been taken into account.

\subsection{Web Interface}

A web interface it has been developed for publication and social network's audiovisual documents access. Although the final and main mission of this part is to 
encourage participation and interaction among network's members, it requires other web 2.0 features which are not implemented yet.

This interface have been developed using Symfony 3 , a web application framework for PHP projects. It is an open source framework with a huge and detailed documentation. This kind of tool will permit a fast grown of the web interface. We will be able to implement more complex features at every version without making serious changes on basis.

Last version of this interface can be tested at the site http://mowento.cs.us.es. Currently, this version permits users login, viewing and searching audiovisual documents and a limited interactions among users. A user can comment the contents, vote both of them, content and comments, as well as he can make his favorite content and user's lists. Features that will permit us a first basic interaction between them. Tagging and post-tagging systems are transparent up to now, because the ontology is centered on academic events, at this moment.

\subsection{Multiagent System}

In order to implement all semantic features on Mowento, we will use a multiagent system, with a restricted set of semantic behaviours. In a first step, this multiagent system will be composed of two types of agents. The first agent group will have some generalized behaviours and it will be responsible for classifying the semantic tasks to execute. The second group will be a very specialized group to perform a few, very specific, semantic tasks. The first specific semantic task will be Post-tagging audiovisual contents, to help users managing its content. A greater repository of semantic behaviours will be created and grow as semantic behaviours are defined.

To support our multiagent system we choose JADE [1] which is an open source middleware under LPGL license. JADE offers a development and living environment as well as a set of libraries that allows to execute and to debug agents. This set of development libraries let us a fast way to implement operatingsystem and device independent agents. These will able us, in the near future, migrate some agents to mobile device and increase the semantic managment of contents at mobile platforms. Also some graphic tools are included to monitoring the agents in real time.

A key issue to using JADE platform is that all implemented protocols are FIPA-compliant and an interaction with other systems are simplified.

Focused on the behaviors, the first specialized one, will be a rule-based behaviour. For such systems, the Jes\& (Java Expert System Shell) rule based engine is advised. Jess is implemented in Java and this will let us to integrate with Jade easily. It was inspired in CLIPS, and it can compile any file build for this. Jess can manage facts, rules and run the inference engine in the same way, too. Also java objects can be handled and this provide us the chance for creating deliberative behaviours and integrate them into agents.

${ }^{3}$ http://www.symfony-project.org/

${ }^{4}$ http://herzberg.ca.sandia.gov/jess/ 
Integration is made following the tutorial written at Jade websit 5 . It starts creating a rule-inference engine and loading all facts and rules from a file. Finally, executing the engine to get all the new facts that can be deduced from original facts and rules.

\subsection{ConExp. Making Rules}

One of the most important elements in the semantic content managmement is ConExp 6. This system is responsible for extracting the rules of deduction, by means of Stem basis, which subsequently become a production system and it will be loaded into a Jade agent.

\section{Semantic Tasks}

The semantic tasks to be realized in this experiment will be executed by a multiagent system, with a multi-role society where some different kind of agents can be found. Our aim is suggesting new knowledge(tags) to mobile-network users, which makes tagging process easier and improve the audiovisual contents organization. This task will be a post-tagging task by the WWW interface and it will be assisted by the multiagent system, in order to improve the features of MW2.0 explained before(see section 2), like managing personal information, collecting and to sharing digital objects.

First of all, we built a concept lattice, by means of attribute exploration, focused on "events" and trying to describe all multimedia content that could be generated by assistants through mobile phone's cameras. Processing this concept lattice with the FCA tool, Conexp, we can obtain the Stem basis associated to this lattice and transforms it into a production system and written in Jess syntax to be inserted into agents. The interpretation of an Stem Basis as a production system is sound, as a Tagging exploration method. It is based on the prior interpretation of Attribute Exploration method, (used as refinement method on Mowento's Ontology) . Roughly speaking, the modified version of attribute exploration changes original attribute exploration questions. Thus, "Every object that has the attribute $X_{1}, \ldots, X_{n}$ has also the attribute $Y$ ?" is replaced by "Every photo/video that has the tags $X_{1}, \ldots, X_{n}$ must to have the tag $Y$ ?". Also we can obtain an new ontology which represents the evolved knowledge, after post-tagging, which is included in the new lattice. (See fig 4).

The multiagent system has a specialized agent, which is the supervisor, for all contents and it plans all semantic tasks (see fig 5). Currently, the task in which we are interested for is "suggesting tags" by means of a deliberetive (rule-based) behaviour. Therefore, when a new content arrives to Mowento's platform, the supervisor creates a new agent to execute the proper semantic behaviour.

\footnotetext{
${ }^{5}$ http://jade.tilab.com/doc/tutorials/jade-jess/jade_jess.html

${ }^{6}$ http://conexp.sourceforge.net/
} 


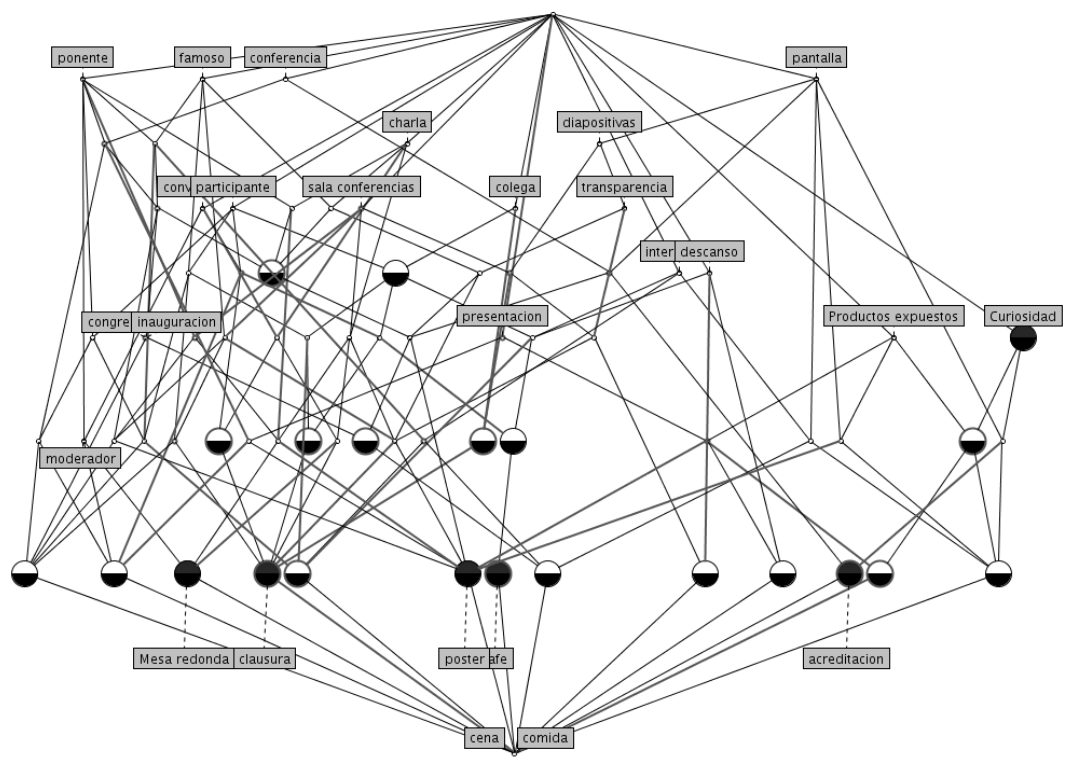

Fig. 4. FCA tool translation: Concept lattice

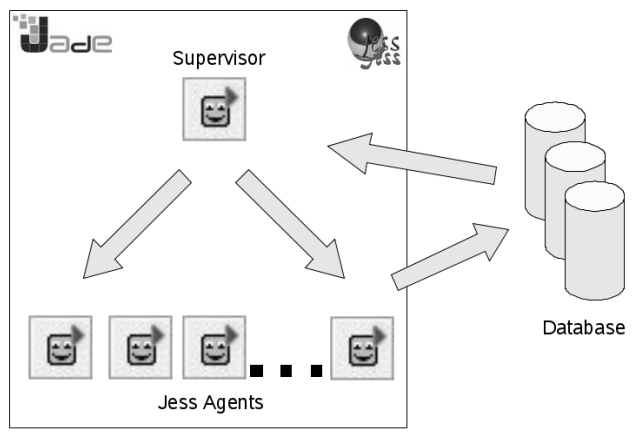

Fig. 5. Agents Architecture

This new agent, read the content from database, load the rules from the Jess file created above and initializes the rule's engine with facts (tags) written by the user. The engine is executed to obtain a new set of facts (tags) which will be inserted into database to be suggested to the user, through the web interface.

Formal Concept Analisys[5] has emerged as an interesting tool for the extraction of formal concepts from labeled audiovisual content. This is our first approach for building a repository of semantic behaviours and get totally semantic mobile social network. 


\section{Conclusions and Future Work}

In this paper we present an application of a Multiagent System to Mobile Web 2.0. The aim of the MAS is the semantic management of user generated content, suggesting and adding semantic metadata through an specific interpretation of STEM basis.

The biggest handicap to use Semantic Web tools on mobile devices is related to usability of these as generator of metadata. This handicap is (partially) solved here, for some extent, using an agent platform monitoring the proper content managment generated by every user. We found that the use of Formal Concept Analysis tools and techniques can be very useful for generation of more sophisticated metadata that the user generates from mobile devices.

Future work is aimed to satisfying other semantic/cognitive requirements mentioned:

- Visual debugging of personal ontologies.

- Expanding, through the FOAF network of the user, the knowledge generated (with the labels suggested).

\section{References}

1. Bellifemine, F., Poggi, A., Rimassa, G.: Jade - a fipa-compliant agent framework. Technical report, CSELT (1999)

2. Borrego-Díaz, J., Chávez-González, A.M.: Visual ontology cleaning: Cognitive principles and applicability. In: Sure, Y., Domingue, J. (eds.) ESWC 2006. LNCS, vol. 4011, pp. 317-331. Springer, Heidelberg (2006)

3. Ganter, B., Wille, R.: Formal Concept Analysis - Mathematical Foundations. Springer, Heidelberg (1999)

4. Jaokar, A., Fish, T.: Mobile Web 2.0: The Innovator's Guide to Developing and Marketing Next Generation Wireless/Mobile Applications. Future Text Pub. (2006)

5. Jäschke, R., Hotho, A., Schmitz, C., Ganter, B., Stumme, G.: Discovering shared conceptualizations in folksonomies. Journal of Web Semantics 6(1), 38-53 (2008)

6. Martignoni, R., Stanoevska-Slabeva, K.: Mobile web 2.0. In: Conference Proceedings of the 20th Bled eConference, pp. 1-18 (2007)

7. Odlyzko, A.: Content is not king. First Monday 6 (2001)

8. Onnela, J.P., Saramaki, J., Hyvonen, J., Szabo, G., Lazer, D., Kaski, K., Kertesz, J., Barabasi, A.L.: Structure and tie strengths in mobile communication networks. Proceedings of the National Academy of Sciences 104(18), 7332-7336 (2007)

9. Smith, G.: Tagging: People-powered Metadata for the Social Web (Voices That Matter). New Riders Press (December 2007)

10. Spivack, N.: New version of my "metaweb" graph - the future of the net. Minding the Planet (2004) 